\title{
Asian Option Pricing with Transaction Costs and Dividends under the Fractional Brownian Motion Model
}

\author{
Yan Zhang, Di Pan, Sheng-Wu Zhou, and Miao Han \\ College of Sciences, China University of Mining and Technology, Jiangsu, Xuzhou 221116, China \\ Correspondence should be addressed to Sheng-Wu Zhou; zswcumt@163.com
}

Received 25 December 2013; Accepted 12 February 2014; Published 26 March 2014

Academic Editor: Nazim I. Mahmudov

Copyright (C) 2014 Yan Zhang et al. This is an open access article distributed under the Creative Commons Attribution License, which permits unrestricted use, distribution, and reproduction in any medium, provided the original work is properly cited.

The pricing problem of geometric average Asian option under fractional Brownian motion is studied in this paper. The partial differential equation satisfied by the option's value is presented on the basis of no-arbitrage principle and fractional formula. Then by solving the partial differential equation, the pricing formula and call-put parity of the geometric average Asian option with dividend payment and transaction costs are obtained. At last, the influences of Hurst index and maturity on option value are discussed by numerical examples.

\section{Introduction}

Option pricing theory has been an unprecedented development since the classic Black-Scholes option pricing model [1] was proposed. Asian options are a kind of common strong path-dependent options, whose value depends on the average price of the underlying asset during the life of the option. Fusai and Meucci [2] have discretely studied Asian option pricing problem under the Levy process. Vecer [3] has got the unified algorithm of the Asian option value based on the basic theory of stochastic analysis. Večeř and Xu [4] extended the method of removing path correlation to the case in a semimartingale model and obtained the partial differential equation of the option value under the standard Brownian motion.

However, the empirical analysis shows that there is a longterm correlation between the underlying asset prices, so that the geometric Brownian motion is not considered as an ideal tool to describe the process of asset price. Since fractional Brownian motion has the properties of self-similarity, thick tail, and long-term correlation, that fractional Brownian motion has become a good tool to depict the process of underlying asset price. According to the standard Brownian motion, Mandelbrot and Van Ness [5] obtained a stochastic integral form of fractional Brownian motion. Based on the wick product, Duncan et al. [6] introduced fractional Itô integral. Elliott and van der Hoek and others [7] have studied fractional Brownian motion with Hurst parameter belonging to the interval $(1 / 2,1)$, and they obtained fractional Girsanov theorem and fractional Itô formula by using wick product. Because an Itô formula for generalized functionals of a fractional Brownian motion with arbitrary Hurst parameter [8] was obtained by Christian Bender, it brought great convenience to option pricing.

In the reality of the securities market, investors were faced with considerable and nonignorable transaction costs and Leland [8] firstly examined the problems of option pricing and hedging with transaction costs. Due to infinite variation of geometric Brownian motion, transaction costs would become infinite in the continuous time completely hedging strategy. So Leland suggested that no-arbitrage assumption is replaced by Delta hedging strategy under the condition of discrete time occasions and transaction costs. The model was then extended by Hoggard et al. and others [9]. Guasoni [10] studied the standard option with transaction costs under the fractional Brownian motion, but he did not obtain option pricing formula. Then Liu and Chang and others [11] extended the option pricing with transaction costs under fractional Brownian motion and provide an approximate solution of the nonlinear HoggardWhalley-Wilmott equation. Wang et al. and others [12-16] have systematically discussed the European option pricing problems with transaction costs and long-range dependence. But these studies are usually aimed at European standard 
options. To the authors' knowledge, there does not exist systematic research about Asian options under time-varying fractional Brownian motion.

In this paper, Asian option pricing problems with transaction costs and dividends under fractional Brownian motion are studied. Firstly, the partial differential equation satisfied by geometric average Asian option value is obtained on the basis of no-arbitrage principle. Then the analytic expressions of option value and parity formula are presented by solving the partial differential equation. At last, the influences of Hurst exponent and maturity on option value are discussed by numerical examples.

\section{Geometric Average Asian Options Pricing Model under Fractional Brownian Motion}

Definition 1 (see [17]). Let $(\Omega, F, P)$ be a complete probability space on which a standard fractional Brownian motion with Hurst exponent $H(0<H<1)$ is continuous, centered Gaussian processes $\left\{B_{H}(t), t \geq 0\right\}$ with covariance functions $\operatorname{Cov}\left(B_{H}(t), B_{H}(s)\right)=(1 / 2)\left(|t|^{2 H}+|s|^{2 H}-|t-s|^{2 H}\right), s, t>0$.

In 2003, Bender has obtained an Itô formula for generalized functionals of a fractional Brownian motion with arbitrary Hurst parameter [18]. The following lemma is obtained by using the integral Itô formula.

Lemma 2. Suppose that stochastic process $S_{t}$ satisfied the following equation:

$$
d S_{t}=\mu_{t} S_{t} d t+\sigma_{t} S_{t} d B_{H}(t)
$$

where $\mu_{t}$ and $\sigma_{t}$ are, respectively, drift coefficient and diffusion coefficient. Suppose that stochastic process $f=f\left(t, J_{t}, S_{t}\right)$; then, for any $t \in[0, T]$, one has

$$
\begin{aligned}
d f= & \left(\frac{\partial f}{\partial t}+\mu_{t} S_{t} \frac{\partial f}{\partial S_{t}}+\frac{\partial f}{\partial J_{t}} \frac{d J_{t}}{d t}+H\left(\sigma_{t} S_{t}\right)^{2} t^{2 H-1} \frac{\partial^{2} f}{\partial S_{t}^{2}}\right) d t \\
& +\sigma_{t} S_{t} \frac{\partial f}{\partial S_{t}} d B_{H}(t),
\end{aligned}
$$

where $J_{t}=e^{(1 / t) \int_{0}^{t} \ln S_{\tau} d \tau}$ is geometric average of $S_{t}$ between the time period of $[0, t]$.

In this paper, the following basic assumptions were needed.

(i) Underlying asset price, $S_{t}$, satisfied the stochastic differential equations

$$
d S_{t}=\left(\mu_{t}-q_{t}\right) S_{t} d t+\sigma S_{t} d B_{H}(t)
$$

where $\mu_{t}$ is the expected return, $q_{t}$ denotes dividend yield, $\sigma$ is volatility, and $B_{H}(t)$ is a fractional Brownian motion.

(ii) Risk-free interest rate $r_{t}$ is a certain function of time $t$. (iii) Transaction costs are proportional to the value of the transaction in the underlying. Let $k$ denote the transaction cost per unit dollar of transaction, where $k$ is a constant. To buy or sell $v_{t}$ shares of the underlying asset need pay proportional transaction costs $\left(k\left|v_{t}\right| S_{t}\right)$; note that $v_{t}>0$ denotes buying the underlying asset and $v_{t}<0$ denotes selling.

(iv) The expected return of the hedge portfolio equals the risk-free rate $r_{t}$.

Let $V=V\left(t, J_{t}, S_{t}\right)$ denote the value of the geometric average Asian call at time $t$, where $J_{t}=e^{(1 / t) \int_{0}^{t} \ln S_{\tau} d \tau}$ is geometric average of underlying asset in $[0, t]$. Construct a portfolio $\Pi$ : long one position of the geometric average Asian call, and sell $\Delta$ shares of the underlying asset. Then the value of the portfolio at time $t$ is

$$
\Pi_{t}=V_{t}-\Delta_{t} S_{t}
$$

After the time interval $\delta t$, the change in the value of the portfolio $\Pi$ is as follows:

$$
\begin{aligned}
\delta \Pi_{t}= & \delta V_{t}-\Delta_{t} \delta S_{t}-\Delta_{t} q_{t} S_{t} \delta t-k\left|v_{t}\right| S_{t+\delta t} \\
= & \left(\frac{\partial V}{\partial t}+H \sigma^{2} S_{t}^{2} t^{2 H-1} \frac{\partial^{2} V}{\partial S_{t}^{2}}-\Delta_{t} q_{t} S_{t}\right) \delta t \\
& +\left(\frac{\partial V}{\partial S_{t}}-\Delta_{t}\right) \delta S_{t}+\frac{\partial V}{\partial J_{t}} \delta J_{t}-k\left|v_{t}\right| S_{t+\delta t}
\end{aligned}
$$

where $\delta S_{t}$ denotes the change in the underlying asset price and $v_{t}=\Delta_{t+\delta t}-\Delta_{t}$ is the change of the underlying asset share in $[t, t+\delta t]$. Choose $\Delta_{t}=\partial V / \partial S_{t}$; then, (5) becomes

$$
\begin{aligned}
\delta \Pi_{t}= & \left(\frac{\partial V}{\partial t}+H \sigma^{2} S_{t}^{2} t^{2 H-1} \frac{\partial^{2} V}{\partial S_{t}^{2}}-\frac{\partial V}{\partial S_{t}} q_{t} S_{t}\right) \delta t \\
& +\frac{\partial V}{\partial J_{t}} \delta J_{t}-k\left|v_{t}\right| S_{t+\delta t}
\end{aligned}
$$

where

$$
\begin{aligned}
v_{t} & =\Delta_{t+\delta t}-\Delta_{t}=\frac{\partial V}{\partial S_{t+\delta t}}-\frac{\partial V}{\partial S_{t}} \\
& =\frac{\partial^{2} V}{\partial S_{t}^{2}} \delta S_{t}+\frac{\partial^{2} V}{\partial S_{t} \partial J_{t}} \delta J_{t}+O(\delta t) \\
& =\frac{\partial^{2} V}{\partial S_{t}^{2}} \sigma S_{t} \delta B_{H}(t)+O(\delta t) .
\end{aligned}
$$

The mathematical expectation of transaction costs is obtained in the following form:

$$
\begin{aligned}
E\left(k\left|v_{t}\right| S_{t+\delta t}\right) & =k\left|\frac{\partial^{2} V}{\partial S_{t}^{2}}\right| \sigma S_{t} E\left(\left|\delta B_{H}(t)\right| S_{t+\delta d}\right)+O(\delta t) \\
& =\sqrt{\frac{2}{\pi}} k \sigma S_{t}^{2}(\delta t)^{H}\left|\frac{\partial^{2} V}{\partial S_{t}^{2}}\right|+O(\delta t) .
\end{aligned}
$$


By assumption (iv), the following relation holds:

$$
E\left(\delta \Pi_{t}\right)=r_{t} \Pi_{t} \delta t
$$

By (6) and (8), one has

$$
\begin{aligned}
E\left(\delta \Pi_{t}\right)= & \left(\frac{\partial V}{\partial t}+H \sigma^{2} S_{t}^{2} t^{2 H-1} \frac{\partial^{2} V}{\partial S_{t}^{2}}-\frac{\partial V}{\partial S_{t}} q_{t} S_{t}\right) \delta t \\
& +\frac{\partial V}{\partial J_{t}} \delta J_{t}-\sqrt{\frac{2}{\pi}} k \sigma S_{t}^{2}(\delta t)^{H}\left|\frac{\partial^{2} V}{\partial S_{t}^{2}}\right|+O(\delta t) .
\end{aligned}
$$

Substituting (10) into (9), the following partial differential equation is obtained:

$$
\begin{aligned}
\frac{\partial V}{\partial t} & +H \sigma^{2} S_{t}^{2} t^{2 H-1} \frac{\partial^{2} V}{\partial S_{t}^{2}}+\left(r_{t}-q_{t}\right) S_{t} \frac{\partial V}{\partial S_{t}}+\frac{\partial V}{\partial J_{t}} \frac{d J_{t}}{d t} \\
& -\sqrt{\frac{2}{\pi}} k \sigma S_{t}^{2}(\delta t)^{H-1}\left|\frac{\partial^{2} V}{\partial S_{t}^{2}}\right|-r_{t} V=0 .
\end{aligned}
$$

Substituting $J_{t}=e^{(1 / t) \int_{0}^{t} \ln S_{\tau} d \tau}$ and $d J_{t} / d t=J_{t} \ln \left(S_{t} / J_{t}\right) / t$ into (11), the following equation is obtained:

$$
\begin{aligned}
\frac{\partial V}{\partial t} & +H \sigma^{2} S_{t}^{2} t^{2 H-1} \frac{\partial^{2} V}{\partial S_{t}^{2}}+\left(r_{t}-q_{t}\right) S_{t} \frac{\partial V}{\partial S_{t}}+\frac{\partial V}{\partial J_{t}} \frac{J_{t} \ln \left(S_{t} / J_{t}\right)}{t} \\
& -\sqrt{\frac{2}{\pi}} k \sigma S_{t}^{2}(\delta t)^{H-1}\left|\frac{\partial^{2} V}{\partial S_{t}^{2}}\right|-r_{t} V=0
\end{aligned}
$$

denoting

$$
\tilde{\sigma}^{2}=2 \sigma^{2}\left(H t^{2 H-1}-\sqrt{\frac{2}{\pi}} \frac{k}{\sigma}(\delta t)^{H-1} \operatorname{sign}\left(V_{S S}\right)\right) .
$$

Substituting (13) into (12), the following result is obtained.

Theorem 3. Suppose that the underlying asset price $S_{t}$ satisfied (3); then, the value of the geometric average Asian call at time $t(0 \leq t \leq T), V\left(t, J_{t}, S_{t}\right)$, satisfies the following mathematical model:

$$
\begin{gathered}
\frac{\partial V}{\partial t}+\frac{1}{2} \widetilde{\sigma}^{2} S_{t}^{2} \frac{\partial^{2} V}{\partial S_{t}^{2}}+\left(r_{t}-q_{t}\right) S_{t} \frac{\partial V}{\partial S_{t}} \\
+\frac{\partial V}{\partial J_{t}} \frac{J_{t} \ln \left(S_{t} / J_{t}\right)}{t}-r_{t} V=0 .
\end{gathered}
$$

Remark 4. Theorem 3 is obtained for the long position of the option. If the short position of option is considered, similarly, we can also get the mathematical model (14) and only the corresponding modified volatility is given by the following form:

$$
\tilde{\sigma}^{2}=2 \sigma^{2}\left(H t^{2 H-1}+\sqrt{\frac{2}{\pi}} \frac{k}{\sigma}(\delta t)^{H-1} \operatorname{sign}\left(V_{S S}\right)\right) .
$$

Let $\operatorname{Le}(H)=\sqrt{2 / \pi}(k / \sigma)(\delta t)^{H-1}$, which is called fractional Leland number [8].
Remark 5. For the long position of a single European Asian option, its final payoff is $\left(J_{T}-K\right)^{+}$or $\left(K-J_{T}\right)^{+}$and they are both convex function, so $V_{J J}>0$, and noticing $J_{t}=$ $e^{(1 / t) \int_{0}^{t} \ln S_{\tau} d \tau}$, thus $V_{S S}>0$. However, for the short position of a single European Asian option, its final payoff at maturity is $-\left(J_{T}-K\right)^{+}$or $-\left(K-J_{T}\right)^{+}$and they are both concave function, so that $V_{J J}<0, V_{S S}<0$. So for a single European Asian option, (13) and (15) can be represented as

$$
\widetilde{\sigma}^{2}=2 \sigma^{2}\left(H t^{2 H-1}-\sqrt{\frac{2}{\pi}} \frac{k}{\sigma}(\delta t)^{H-1}\right)
$$

\section{Option Pricing Formula}

Theorem 6. Suppose that the underlying asset price $S_{t}$ satisfied (3); then, the value, $V\left(t, J_{t}, S_{t}\right)$, of the geometric average Asian call with strike price $K$, maturity $T$, and transaction fee rate $k$ at time $t$ is

$$
\begin{aligned}
V\left(t, J_{t}, S_{t}\right)= & \left(J_{t}^{t} S_{t}^{T-t}\right)^{1 / T} e^{r^{*}(T-t)-\int_{t}^{T} r_{\theta} d \theta+\left(\sigma^{* 2} / 2\right)\left(T^{2 H}-t^{2 H}\right)} N\left(d_{1}\right) \\
& -K e^{-\int_{t}^{T} r_{\theta} d \theta} N\left(d_{2}\right),
\end{aligned}
$$

where

$$
\begin{aligned}
& d_{1}=\frac{\ln \left[\left(J_{t}^{t} S_{t}^{T-t}\right)^{1 / T} / K\right]+r^{*}(T-t)+\sigma^{* 2}\left(T^{2 H}-t^{2 H}\right)}{\sigma^{*} \sqrt{T^{2 H}-t^{2 H}}}, \\
& d_{2}=d_{1}-\sigma^{*} \sqrt{T^{2 H}-t^{2 H}} \text {, } \\
& r^{*}=\frac{\int_{t}^{T}\left(r_{\theta}-q_{\theta}\right)((T-\theta) / T) d \theta}{T-t}-\frac{\sigma^{2}\left(T^{2 H}-t^{2 H}\right)}{2(T-t)} \\
& +\frac{H \sigma^{2}\left(T^{2 H+1}-t^{2 H+1}\right)}{T(2 H+1)(T-t)}+\frac{1}{2} \operatorname{Le}(H) \sigma^{2} \frac{T-t}{T}, \\
& \sigma^{*}=\sigma \times\left(1-\frac{4 H\left(T^{2 H+1}-t^{2 H+1}\right)}{T(2 H+1)\left(T^{2 H}-t^{2 H}\right)}\right. \\
& +\frac{H\left(T^{2 H+2}-t^{2 H+2}\right)}{T^{2}(H+1)\left(T^{2 H}-t^{2 H}\right)} \\
& \left.-2 \operatorname{Le}(H) \frac{(T-t)^{3}}{3 T^{2}\left(T^{2 H}-t^{2 H}\right)}\right)^{1 / 2} \text {, } \\
& N(x)=\int_{-\infty}^{x} \frac{1}{\sqrt{2 \pi}} e^{-t^{2} / 2} d t .
\end{aligned}
$$


Proof. By Theorem 3 , the value, $V\left(t, J_{t}, S_{t}\right)$, of the geometric average Asian call satisfies the following model:

$$
\begin{gathered}
\frac{\partial V}{\partial t}+\frac{1}{2} \widetilde{\sigma}^{2} S_{t}^{2} \frac{\partial^{2} V}{\partial S_{t}^{2}}+\left(r_{t}-q_{t}\right) S_{t} \frac{\partial V}{\partial S_{t}} \\
+\frac{\partial V}{\partial J_{t}} \frac{J_{t} \ln \left(S_{t} / J_{t}\right)}{t}-r_{t} V=0 \\
V\left(T, J_{T}, S_{T}\right)=\left(J_{T}-K\right)^{+} .
\end{gathered}
$$

Let $\xi_{t}=(1 / T)\left[t \ln J_{t}+(T-t) \ln S_{t}\right], V\left(t, J_{t}, S_{t}\right)=U\left(t, \xi_{t}\right)$; then

$$
\begin{gathered}
\frac{\partial V}{\partial t}=\frac{\ln \left(J_{t} / S_{t}\right)}{T} \frac{\partial U}{\partial \xi_{t}}+\frac{\partial U}{\partial t}, \quad \frac{\partial V}{\partial S_{t}}=\frac{T-t}{T S_{t}} \frac{\partial U}{\partial \xi_{t}}, \\
\frac{\partial^{2} V}{\partial S_{t}^{2}}=\left(\frac{T-t}{T S_{t}}\right)^{2} \frac{\partial^{2} U}{\partial \xi_{t}^{2}}-\frac{T-t}{T S_{t}^{2}} \frac{\partial U}{\partial \xi_{t}}, \quad \frac{\partial V}{\partial J_{t}}=\frac{t}{T J_{t}} \frac{\partial U}{\partial \xi_{t}} .
\end{gathered}
$$

Combined with the boundary conditions of the call option, $V\left(T, J_{T}, S_{T}\right)=\left(J_{T}-K\right)^{+}$, the model (19) can be converted to

$$
\begin{gathered}
\frac{\partial U}{\partial t}+\left(r_{t}-q_{t}-\frac{\widetilde{\sigma}^{2}}{2}\right) \frac{T-t}{T} \frac{\partial U}{\partial \xi_{t}}+\frac{\widetilde{\sigma}^{2}}{2}\left(\frac{T-t}{T}\right)^{2} \frac{\partial^{2} U}{\partial \xi_{t}^{2}} \\
-r_{t} U=0, \\
U\left(\xi_{T}, T\right)=\left(e^{\xi_{T}}-K\right)^{+} .
\end{gathered}
$$

Let $\tau=\gamma(t), \eta_{\tau}=\xi_{t}+\alpha(t), W\left(\tau, \eta_{\tau}\right)=U\left(t, \xi_{t}\right) e^{\beta(t)}$ which satisfied the conditions

$$
\alpha(T)=\beta(T)=\gamma(T)=0,
$$

and then we have

$$
\begin{aligned}
& \frac{\partial U}{\partial t}=e^{-\beta(t)}\left(\frac{\partial W}{\partial \tau} \gamma^{\prime}(t)-\beta^{\prime}(t) W+\frac{\partial W}{\partial \eta_{\tau}} \alpha^{\prime}(t)\right), \\
& \frac{\partial U}{\partial \xi_{t}}=e^{-\beta(t)} \frac{\partial W}{\partial \eta_{\tau}}, \quad \frac{\partial^{2} U}{\partial \xi_{t}^{2}}=e^{-\beta(t)} \frac{\partial^{2} W}{\partial \eta_{\tau}^{2}} .
\end{aligned}
$$

Substituting (23) into (21), we can get

$$
\begin{aligned}
\gamma^{\prime}(t) & \frac{\partial W}{\partial \tau}+\frac{\widetilde{\sigma}^{2}}{2}\left(\frac{T-t}{T}\right)^{2} \frac{\partial^{2} W}{\partial \eta_{\tau}^{2}} \\
+ & {\left[\left(r_{t}-q_{t}-\frac{\widetilde{\sigma}^{2}}{2}\right) \frac{T-t}{T}+\alpha^{\prime}(t)\right] \frac{\partial W}{\partial \eta_{\tau}} } \\
& -\left(r_{t}+\beta^{\prime}(t)\right) W=0 .
\end{aligned}
$$

Set

$$
\begin{gathered}
\left(r_{t}-q_{t}-\frac{\widetilde{\sigma}^{2}}{2}\right) \frac{T-t}{T}+\alpha^{\prime}(t)=0, \quad r_{t}+\beta^{\prime}(t)=0, \\
\gamma^{\prime}(t)=-\frac{\widetilde{\sigma}^{2}}{2}\left(\frac{T-t}{T}\right)^{2} .
\end{gathered}
$$

Combining with the terminal conditions $\alpha(T)=\beta(T)=$ $\gamma(T)=0$, we have

$$
\begin{aligned}
\alpha(t) & =\int_{t}^{T}\left(r_{\theta}-q_{\theta}\right) \frac{T-\theta}{T} d \theta-\int_{t}^{T} \frac{1}{2} \widetilde{\sigma}^{2}\left(\frac{T-\theta}{T}\right) d \theta \\
& =r *(T-t), \\
\beta(t) & =\int_{t}^{T} r_{\theta} d \theta, \\
\gamma(t) & =\int_{t}^{T} \frac{1}{2} \widetilde{\sigma}^{2}\left(\frac{T-\theta}{T}\right)^{2} d \theta=\frac{\sigma^{* 2}}{2}\left(T^{2 H}-t^{2 H}\right),
\end{aligned}
$$

where

$$
\begin{gathered}
r^{*}=\frac{\int_{t}^{T}\left(r_{\theta}-q_{\theta}\right)((T-\theta) / T) d \theta}{T-t}-\frac{\sigma^{2}\left(T^{2 H}-t^{2 H}\right)}{2(T-t)} \\
+\frac{H \sigma^{2}\left(T^{2 H+1}-t^{2 H+1}\right)}{T(2 H+1)(T-t)}+\frac{1}{2} \operatorname{Le}(H) \sigma^{2} \frac{T-t}{T}, \\
\sigma^{*}=\sigma \times\left(1-\frac{4 H\left(T^{2 H+1}-t^{2 H+1}\right)}{T(2 H+1)\left(T^{2 H}-t^{2 H}\right)}\right. \\
\quad+\frac{H\left(T^{2 H+2}-t^{2 H+2}\right)}{T^{2}(H+1)\left(T^{2 H}-t^{2 H}\right)} \\
\left.-2 \operatorname{Le}(H) \frac{(T-t)^{3}}{3 T^{2}\left(T^{2 H}-t^{2 H}\right)}\right)^{1 / 2} .
\end{gathered}
$$

Thus the model (21) is converted into the classic heat conduction equation

$$
\begin{gathered}
\frac{\partial W}{\partial \tau}=\frac{\partial^{2} W}{\partial \eta_{\tau}^{2}}, \\
W\left(\eta_{0}, 0\right)=\left(e^{\eta_{0}}-K\right)^{+} .
\end{gathered}
$$

Its solution is

$$
\begin{aligned}
W\left(\eta_{\tau}, \tau\right) & =\frac{1}{2 \sqrt{\pi \tau}} \int_{-\infty}^{+\infty}\left(e^{y}-K\right)^{+} e^{-\left(y-\eta_{\tau}\right)^{2} / 4 \tau} d y \\
& =\frac{1}{2 \sqrt{\pi \tau}} \int_{\ln K}^{+\infty}\left(e^{y}-K\right) e^{-\left(y-\eta_{\tau}\right)^{2} / 4 \tau} d y \\
& =e^{\eta_{\tau}+\tau} N\left(\frac{2 \tau+\eta_{\tau}-\ln K}{\sqrt{2 \tau}}\right)-K N\left(\frac{\eta_{\tau}-\ln K}{\sqrt{2 \tau}}\right) .
\end{aligned}
$$

After variable reduction, we have

$$
\begin{aligned}
W\left(\eta_{\tau}, \tau\right)= & \left(J_{t}^{t} S_{t}^{T-t}\right)^{1 / T} e^{r^{*}(T-t)+\left(\sigma^{* 2} / 2\right)\left(T^{2 H}-t^{2 H}\right)} N\left(d_{1}\right) \\
& -K N\left(d_{2}\right)
\end{aligned}
$$


where

$$
\begin{aligned}
\frac{2 \tau+\eta_{\tau}-\ln K}{\sqrt{2 \tau}}=\left(\ln \left[\frac{\left(J_{t}^{t} S_{t}^{T-t}\right)^{1 / T}}{K}\right]+r^{*}(T-t)\right. \\
\left.+\sigma^{* 2}\left(T^{2 H}-t^{2 H}\right)\right) \\
\\
\times\left(\sigma^{*}\left(T^{2 H}-t^{2 H}\right)^{1 / 2}\right)^{-1} \triangleq d_{1}, \\
\frac{\eta_{\tau}-\ln K}{\sqrt{2 \tau}}=\frac{\ln \left[\left(J_{t}^{t} S_{t}^{T-t}\right)^{1 / T} / K\right]+r^{*}(T-t)}{\sigma^{*} \sqrt{T^{2 H}-t^{2 H}}} \\
=d_{1}-\sigma^{*} \sqrt{T^{2 H}-t^{2 H}} \triangleq d_{2} .
\end{aligned}
$$

So the value of geometric average Asian call option at time $t$ is obtained

$$
\begin{aligned}
V\left(t, J_{t}, S_{t}\right)= & U\left(\xi_{t}, t\right)=W\left(\eta_{\tau}, \tau\right) e^{-\beta(t)} \\
= & \left(J_{t}^{t} S_{t}^{T-t}\right)^{1 / T} e^{r^{*}(T-t)-\int_{t}^{T} r_{\theta} d \theta+\left(\sigma^{* 2} / 2\right)\left(T^{2 H}-t^{2 H}\right)} N\left(d_{1}\right) \\
& -K e^{-\int_{t}^{T} r_{\theta} d \theta} N\left(d_{2}\right) .
\end{aligned}
$$

Theorem 7. Suppose the underlying asset price $S_{t}$ satisfies (3); then the relationship between $V_{C}\left(t, J_{t}, S_{t}\right)$, the value of geometric average Asian call option, and $V_{P}\left(t, J_{t}, S_{t}\right)$, the value of put option with strike price $K$, maturity $T$, and transaction fee rate $k$ at time $t$, is

$$
\begin{aligned}
V_{C}\left(t, J_{t}, S_{t}\right)-V_{P}\left(t, J_{t}, S_{t}\right) \\
=e^{r^{*}(T-t)-\int_{t}^{T} r_{\theta} d \theta+\left(\sigma^{* 2} / 2\right)\left(T^{2 H}-t^{2 H}\right)} J_{t}^{t / T} S_{t}^{(T-t) / T} \\
\quad-K e^{-\int_{t}^{T} r_{\theta} d \theta},
\end{aligned}
$$

where $r^{*}, \sigma^{*}$ are the same as above.

Proof. Let

$$
W\left(t, J_{t}, S_{t}\right)=V_{C}\left(t, J_{t}, S_{t}\right)-V_{P}\left(t, J_{t}, S_{t}\right) .
$$

Then $W$ is suitable for the following terminal question in $\{0 \leq$ $S<\infty, 0 \leq J<\infty, 0 \leq t \leq T\}$ :

$$
\begin{gathered}
\frac{\partial W}{\partial t}+\left(r_{t}-q_{t}\right) S_{t} \frac{\partial W}{\partial S_{t}}+\frac{\widetilde{\sigma}^{2}}{2} S_{t}^{2} \frac{\partial^{2} W}{\partial S_{t}^{2}} \\
+\frac{\partial W}{\partial J_{t}} J_{t} \frac{\ln S_{t}-\ln J_{t}}{t}-r_{t} W=0, \\
\left.W\right|_{t=T}=\left(J_{T}-K\right)^{+}-\left(K-J_{T}\right)^{+}=J_{T}-K .
\end{gathered}
$$

We let $\xi_{t}=(1 / T)\left[t \ln J_{t}+(T-t) \ln S_{t}\right]$; then $W$ is suitable for the following in $\left\{\xi_{t} \in R, 0 \leq t \leq T\right\}$ :

$$
\begin{gathered}
\frac{\partial W}{\partial t}+\left(r_{t}-q_{t}-\frac{\widetilde{\sigma}^{2}}{2}\right) \frac{T-t}{T} \frac{\partial W}{\partial \xi_{t}} \\
+\frac{\widetilde{\sigma}^{2}}{2}\left(\frac{T-t}{T}\right)^{2} \frac{\partial^{2} W}{\partial \xi_{t}^{2}}-r W=0, \\
\left.W\right|_{t=T}=e^{\xi_{T}}-K .
\end{gathered}
$$

Set the form solution of problem of (36) is

$$
W\left(t, J_{t}, S_{t}\right)=a(t) e^{\xi_{t}}+b(t) .
$$

Substituting (37) into (36) and comparing coefficients, one has

$$
\begin{gathered}
\left(r_{t}-q_{t}-\frac{\widetilde{\sigma}^{2}}{2}\right) \frac{T-t}{T} a(t)+\frac{\widetilde{\sigma}^{2}}{2}\left(\frac{T-t}{T}\right)^{2} a(t) \\
+a^{\prime}(t)-r_{t} a(t)=0, \\
b^{\prime}(t)-r_{t} b(t)=0 .
\end{gathered}
$$

Taking $a(T)=1, b(T)=-K$, the solutions of (38) are

$$
\begin{aligned}
a(t) & =e^{\alpha(t)-\int_{t}^{T} r_{\tau} d \tau+\gamma(t)} \\
& =e^{r^{*}(T-t)-\int_{t}^{T} r_{\tau} d \tau+\left(\sigma^{* 2} / 2\right)\left(T^{2 H}-t^{2 H}\right)}, \\
b(t) & =-K e^{-\int_{t}^{T} r_{\theta} d_{\theta} .}
\end{aligned}
$$

Thus

$$
\begin{aligned}
W= & a(t) e^{\xi_{t}}+b(t) \\
= & e^{r^{*}(T-t)-\int_{t}^{T} r_{\theta} d \theta+\left(\sigma^{* 2} / 2\right)\left(T^{2 H}-t^{2 H}\right)} J^{t / T} S^{(T-t) / T} \\
& -K e^{-\int_{t}^{T} r_{\theta} d \theta} .
\end{aligned}
$$

The parity formula between call option and put option is

$$
\begin{aligned}
V_{C}\left(t, J_{t}, S_{t}\right)-V_{P}\left(t, J_{t}, S_{t}\right) \\
=e^{r^{*}(T-t)-\int_{t}^{T} r_{\theta} d \theta+\left(\sigma^{* 2} / 2\right)\left(T^{2 H}-t^{2 H}\right)} J_{t}^{t / T} S_{t}^{(T-t) / T} \\
\quad-K e^{-\int_{t}^{T} r_{\theta} d \theta} .
\end{aligned}
$$

Pricing formula of the geometric average Asian put options can be obtained by Theorems 6 and 7 .

Theorem 8. Suppose $S_{t}$ satisfies (3); then the value, $V_{P}\left(t, J_{t}\right.$, $\left.S_{t}\right)$, of the geometric average Asian put option with strike price $K$, maturity $T$, and transaction fee rate $k$ at time $t$ is

$$
\begin{aligned}
V_{P}\left(t, J_{t}, S_{t}\right)= & -\left(J_{t}^{t} S_{t}^{T-t}\right)^{1 / T} e^{r^{*}(T-t)-\int_{t}^{T} r_{\theta} d \theta+\left(\sigma^{* 2} / 2\right)\left(T^{2 H}-t^{2 H}\right)} \\
& \times N\left(-d_{1}\right)+K e^{-\int_{t}^{T} r_{\theta} d \theta} N\left(-d_{2}\right) .
\end{aligned}
$$


In particular, if $H=1 / 2, k=0, r, q, \sigma$ are all constant and price formula (17) is reduced to the following formula [19]:

$$
\begin{aligned}
V\left(t, J_{t}, S_{t}\right)= & \left(J_{t}^{t} S_{t}^{T-t}\right)^{1 / T} e^{\left(r^{*}+\sigma^{* 2} / 2-r\right)(T-t)} N\left(d_{1}\right) \\
& -K e^{-r(T-t)} N\left(d_{2}\right),
\end{aligned}
$$

where

$$
\begin{aligned}
& d_{1}=\frac{\ln \left[\left(J_{t}^{t} S_{t}^{T-t}\right)^{1 / T} / K\right]+\left(r^{*}+\sigma^{* 2}\right)(T-t)}{\sigma^{*} \sqrt{T-t}}, \\
& d_{2}=d_{1}-\sigma^{*} \sqrt{T-t}, \\
& r^{*}=\left(r-q-\frac{\sigma^{2}}{2}\right) \frac{T-t}{2 T}, \quad \sigma^{*}=\sigma \frac{(T-t)}{\sqrt{3} T} .
\end{aligned}
$$

The following results are consequences of the above theorems.

Corollary 9. If risk-free interest rate $r$, dividend yield $q$, and volatility $\sigma$ are all constant, then the price formulas of geometric average Asian call and put option with strike price K, maturity $T$, and transaction fee rate $k$ under fractional Brownian motion at time $t$ are, respectively,

$$
\begin{aligned}
V_{C}\left(t, J_{t}, S_{t}\right)= & \left(J_{t}^{t} S_{t}^{T-t}\right)^{1 / T} e^{\left(r^{*}-r\right)(T-t)+\left(\sigma^{* 2} / 2\right)\left(T^{2 H}-t^{2 H}\right)} N\left(d_{1}\right) \\
& -K e^{-r(T-t)} N\left(d_{2}\right), \\
V_{P}\left(t, J_{t}, S_{t}\right)= & -\left(J_{t}^{t} S_{t}^{T-t}\right)^{1 / T} e^{\left(r^{*}-r\right)(T-t)+\left(\sigma^{* 2} / 2\right)\left(T^{2 H}-t^{2 H}\right)} \\
& \times N\left(-d_{1}\right)+K e^{-r(T-t)} N\left(-d_{2}\right),
\end{aligned}
$$

where

$$
\begin{aligned}
r^{*}= & \frac{(r-q)}{2 T}(T-t)-\frac{\sigma^{2}\left(T^{2 H}-t^{2 H}\right)}{2(T-t)} \\
& +\frac{H \sigma^{2}\left(T^{2 H+1}-t^{2 H+1}\right)}{T(2 H+1)(T-t)}+\frac{1}{2} L e(H) \sigma^{2} \frac{T-t}{T} .
\end{aligned}
$$

The rest of symbols are the same as Theorem 6.

Noticing that if $H=1 / 2, B_{H}(t)$ is standard Brownian motion $B(t)$, the corresponding underlying asset price follows geometric Brownian motion, one has the following results.

Corollary 10. If risk-free interest rate $r_{t}$ and dividend yield $q_{t}$ are the functions of time $t$ and volatility $\sigma$ is constant, then the price formula $V\left(t, J_{t}, S_{t}\right)$ with respect to geometric average Asian call option with strike price $K$, maturity $T$, and transaction fee rate $k$ under standard Brownian motion at time $t$ is

$$
\begin{aligned}
V\left(t, J_{t}, S_{t}\right)= & \left(J_{t}^{t} S_{t}^{T-t}\right)^{1 / T} e^{\left(r^{*}+\sigma^{* 2} / 2\right)(T-t)-\int_{t}^{T} r_{\theta} d \theta} N\left(d_{1}\right) \\
& -K e^{-\int_{t}^{T} r_{\theta} d \theta} N\left(d_{2}\right),
\end{aligned}
$$

where

$$
\begin{aligned}
d_{1}= & \frac{\ln \left[\left(J_{t}^{t} S_{t}^{T-t}\right)^{1 / T} / K\right]+\left(r^{*}+\sigma^{* 2}\right)(T-t)}{\sigma^{*} \sqrt{T-t}}, \\
d_{2}= & d_{1}-\sigma^{*} \sqrt{T-t}, \\
L e\left(\frac{1}{2}\right)= & \sqrt{\frac{2}{\pi} \frac{k}{\sigma}(\delta t)^{-1 / 2}} \\
r^{*}= & \frac{\int_{t}^{T}\left(r_{\theta}-q_{\theta}\right)((T-\theta) / T) d \theta}{T-t} \\
& -\frac{\sigma^{2}(T-t)}{4 T}+\frac{1}{2} \operatorname{Le}\left(\frac{1}{2}\right) \sigma^{2} \frac{T-t}{T} \\
\sigma^{*}= & \sigma \frac{(T-t)}{\sqrt{3} T} \sqrt{1-2 L e\left(\frac{1}{2}\right)} .
\end{aligned}
$$

\section{Numerical Example}

We discuss the impact of Hurst index and transaction rates on the Asian option value by numerical examples. Assume that the parameter selection is as follows:

$$
\begin{array}{crrr}
S_{t}=80, & t=0, & T=1, \\
r=0.05, & q=0.01, & \sigma & =0.4, \\
K=80, & k=0.003, & \delta t=0.02 .
\end{array}
$$

We calculate the value of the option by using the price formula of (45). The relationships between the value of call option or put option and the underlying asset price with different Hurst index are given in Figures 1 and 2, respectively. From Figures 1 and 2, the relationship between Hurst index and Asian option value is negative. Furthermore, the impact on the call option value decreases with the increase of the underlying asset price, but the impact on the put option value decreases with the decrease of the underlying asset price.

By Figures 3 and 4, we can get the change trend of the value of Asian call and put options with the change of maturity and Hurst index at the same time. The option value increases with the maturity increases, but the value of the call option increases faster than the value of a put option increase.

\section{Conclusions}

Asian options are popular financial derivatives that play an essential role in financial market. Pricing them efficiently and accurately is very important both in theory and practice. We have investigated geometric average Asian option valuation problems with transaction costs under the fractional Brownian motion. By no-arbitrage principle and fractional Itô's formula, this paper has deduced PDE satisfied by the option's value. Meanwhile, the pricing formula and call-put parity of the geometric average Asian option with transaction costs are derived by solving PDE. At last, the influences of Hurst exponent and maturity on option value are discussed through numerical examples. 


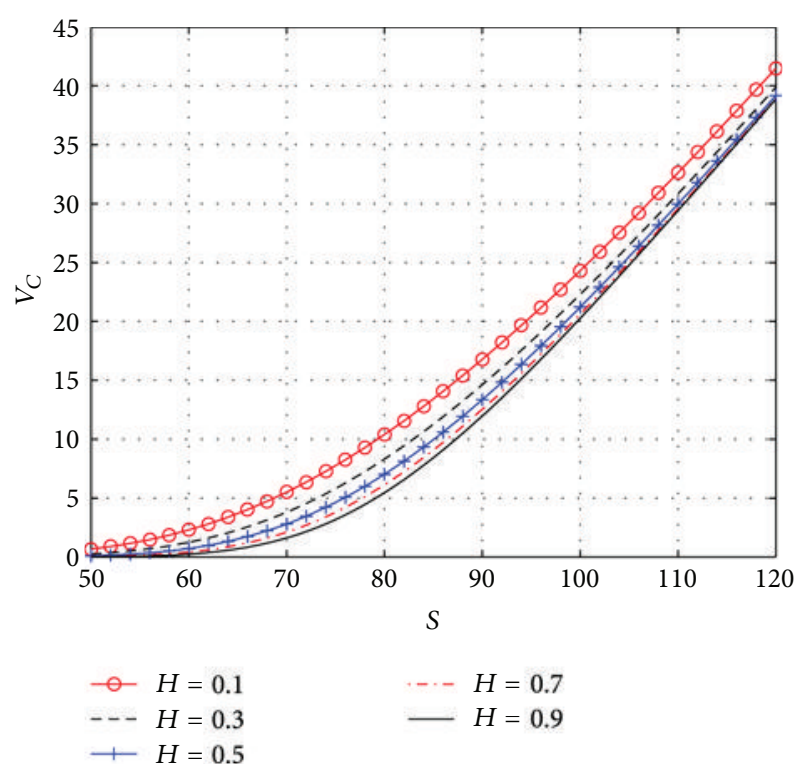

FIgURE 1: The values of the call option with different $H$.

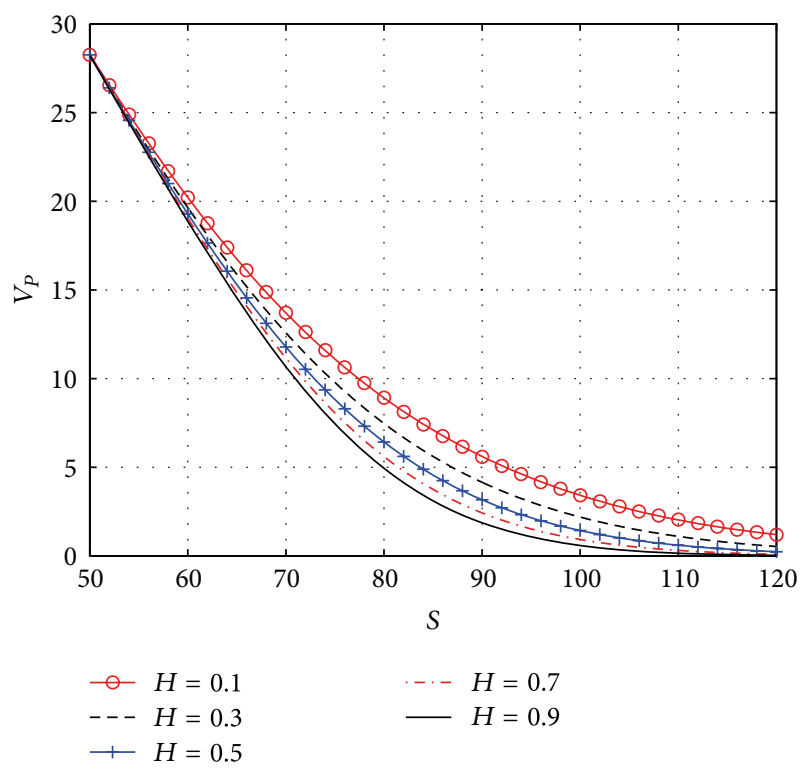

Figure 2: The values of the put option with different $H$.

\section{Conflict of Interests}

The authors declare that there is no conflict of interests regarding the publication of this paper.

\section{Acknowledgments}

The authors would like to thank the anonymous referees for valuable suggestions for the improvement of this paper. All remaining errors are the responsibility of the authors. This research was supported by "the Fundamental Research Funds for the Central Universities" (2010 LKSX03).

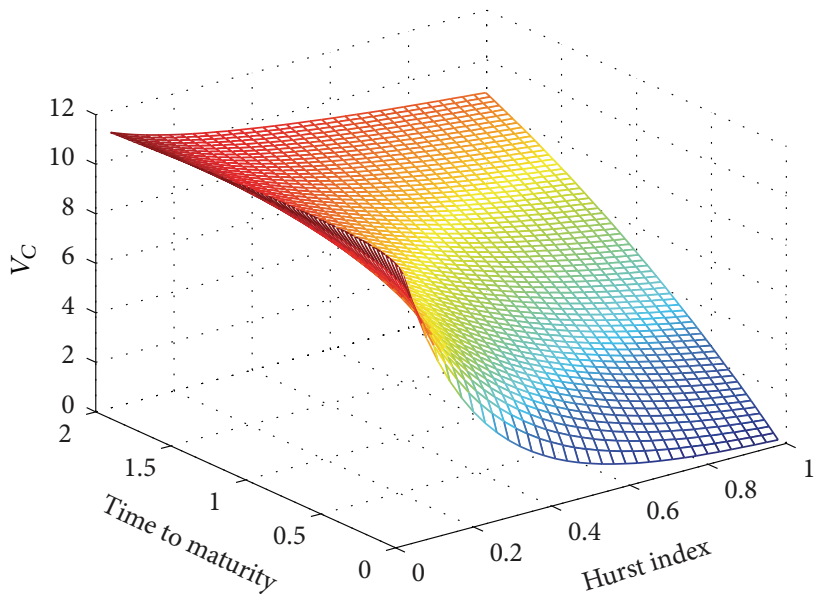

FIGURE 3: Relationship among Hurst index, maturity, and call option value.

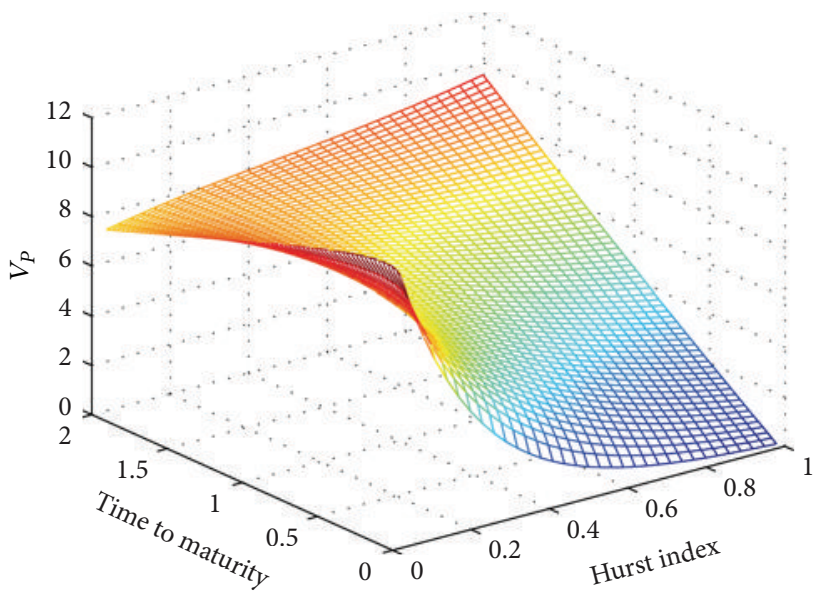

FIGURE 4: Relationship among Hurst index, maturity, and put option value.

\section{References}

[1] F. Black and M. S. Scholes, "The pricing of option and corporate liabilities," Journal of Political Economy, vol. 81, pp. 637-659, 1973.

[2] G. Fusai and A. Meucci, "Pricing discretely monitored Asian options under Lévy processes," Journal of Banking and Finance, vol. 32, no. 10, pp. 2076-2088, 2008.

[3] J. Vecer, "Unified pricing of Asian options," Risk, vol. 6, no. 15, pp. 113-116, 2002.

[4] J. Večeř and M. Xu, "Pricing Asian options in a semimartingale model," Quantitative Finance, vol. 4, no. 2, pp. 170-175, 2004.

[5] B. B. Mandelbrot and J. W. Van Ness, "Fractional Brownian motions, fractional noises and applications," SIAM Review, vol. 10, no. 4, pp. 422-437, 1968.

[6] T. E. Duncan, Y. Hu, and B. Pasik-Duncan, "Stochastic calculus for fractional Brownian motion. I. Theory," SIAM Journal on Control and Optimization, vol. 38, no. 2, pp. 582-612, 2000.

[7] R. J. Elliott and J. van der Hoek, "A general fractional white noise theory and applications to finance," Mathematical Finance, vol. 13, no. 2, pp. 301-330, 2003. 
[8] H. E. Leland, "Option pricing and replication with transactions costs," The Journal of Finance, vol. 40, pp. 1283-1301, 1985.

[9] T. Hoggard, A. E. Whalley, and P. Wilmott, "Hedging option portfolios in the presence of transaction costs," Advanced Futures and Options Research, vol. 7, pp. 21-35, 1994.

[10] P. Guasoni, "No arbitrage under transaction costs, with fractional Brownian motion and beyond," Mathematical Finance, vol. 16, no. 3, pp. 569-582, 2006.

[11] H.-K. Liu and J.-J. Chang, "A closed-form approximation for the fractional Black-Scholes model with transaction costs," Computers \& Mathematics with Applications, vol. 65, no. 11, pp. 1719-1726, 2013.

[12] X.-T. Wang, "Scaling and long-range dependence in option pricing I: pricing European option with transaction costs under the fractional Black-Scholes model," Physica A, vol. 389, no. 3, pp. 438-444, 2010.

[13] X.-T. Wang, E.-H. Zhu, M.-M. Tang, and H.-G. Yan, "Scaling and long-range dependence in option pricing II: pricing European option with transaction costs under the mixed Brownianfractional Brownian model," Physica A, vol. 389, no. 3, pp. 445451, 2010.

[14] X.-T. Wang, H.-G. Yan, M.-M. Tang, and E.-H. Zhu, "Scaling and long-range dependence in option pricing III: a fractional version of the Merton model with transaction costs," Physica A, vol. 389, no. 3, pp. 452-458, 2010.

[15] X.-T. Wang, "Scaling and long range dependence in option pricing, IV: pricing European options with transaction costs under the multifractional Black-Scholes model," Physica A, vol. 389, no. 4, pp. 789-796, 2010.

[16] X.-T. Wang, M. Wu, Z.-M. Zhou, and W.-S. Jing, "Pricing European option with transaction costs under the fractional long memory stochastic volatility model," Physica A, vol. 391, no. 4, pp. 1469-1480, 2012.

[17] Y. Hu and B. Øksendal, "Fractional white noise calculus and applications to finance," Infinite Dimensional Analysis, Quantum Probability and Related Topics, vol. 6, no. 1, pp. 1-32, 2003.

[18] C. Bender, "An Itô formula for generalized functionals of a fractional Brownian motion with arbitrary Hurst parameter," Stochastic Processes and Their Applications, vol. 104, no. 1, pp. 81-106, 2003.

[19] J. Li-Shang, Mathematical Modeling and Methods of Option Pricing, Higher Education Press, Beijing, China, 2nd edition, 2008. 


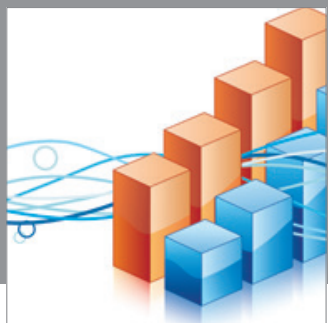

Advances in

Operations Research

mansans

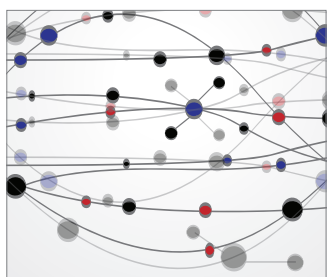

The Scientific World Journal
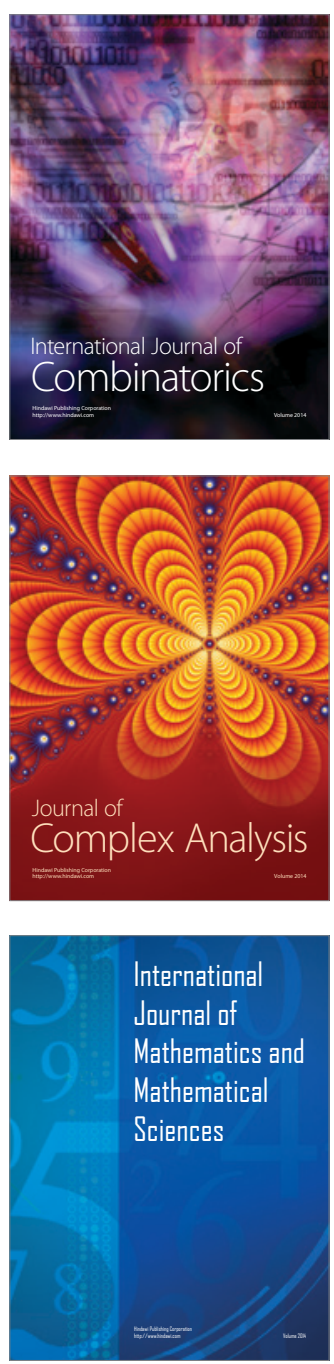
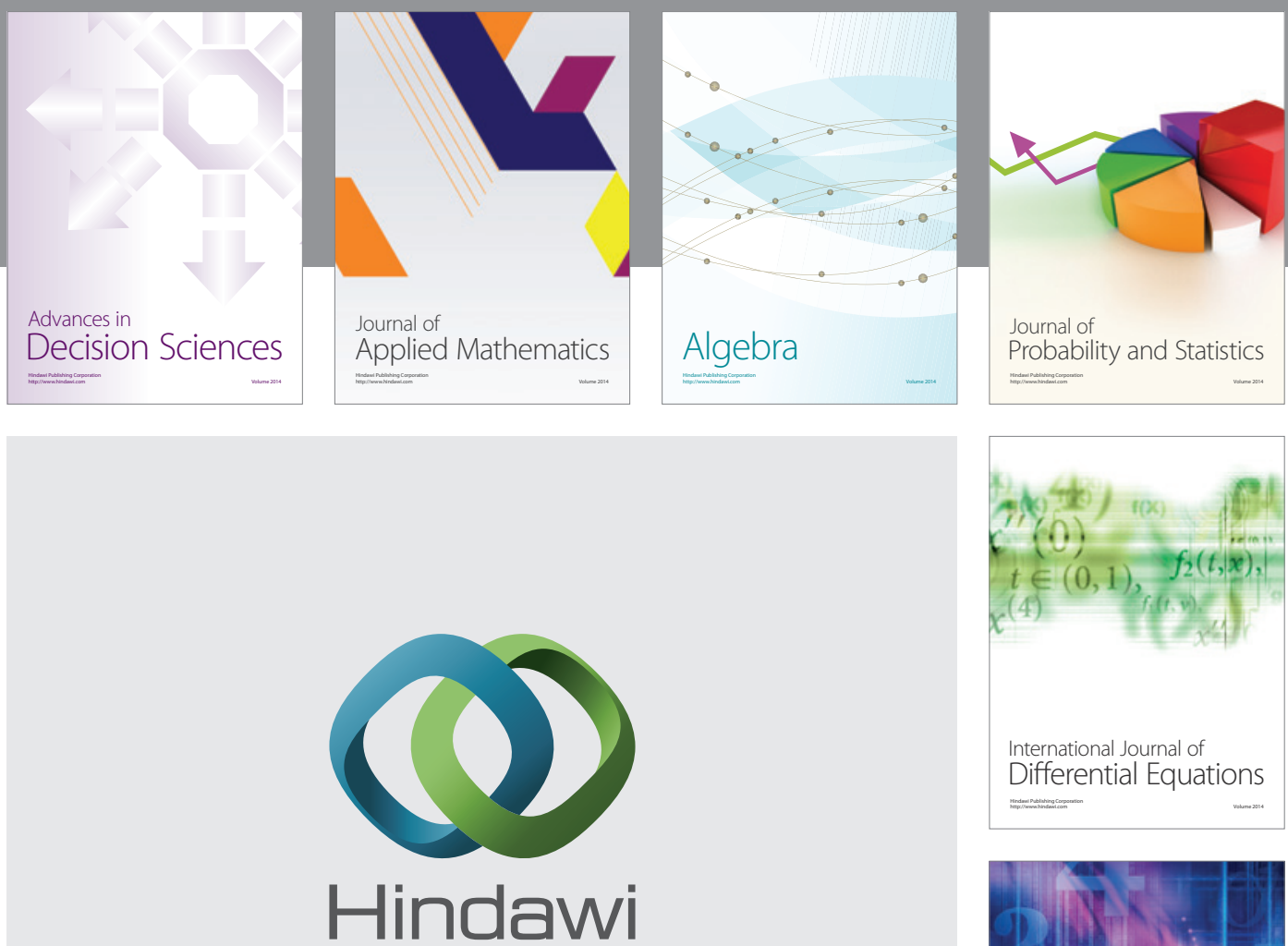

Submit your manuscripts at http://www.hindawi.com
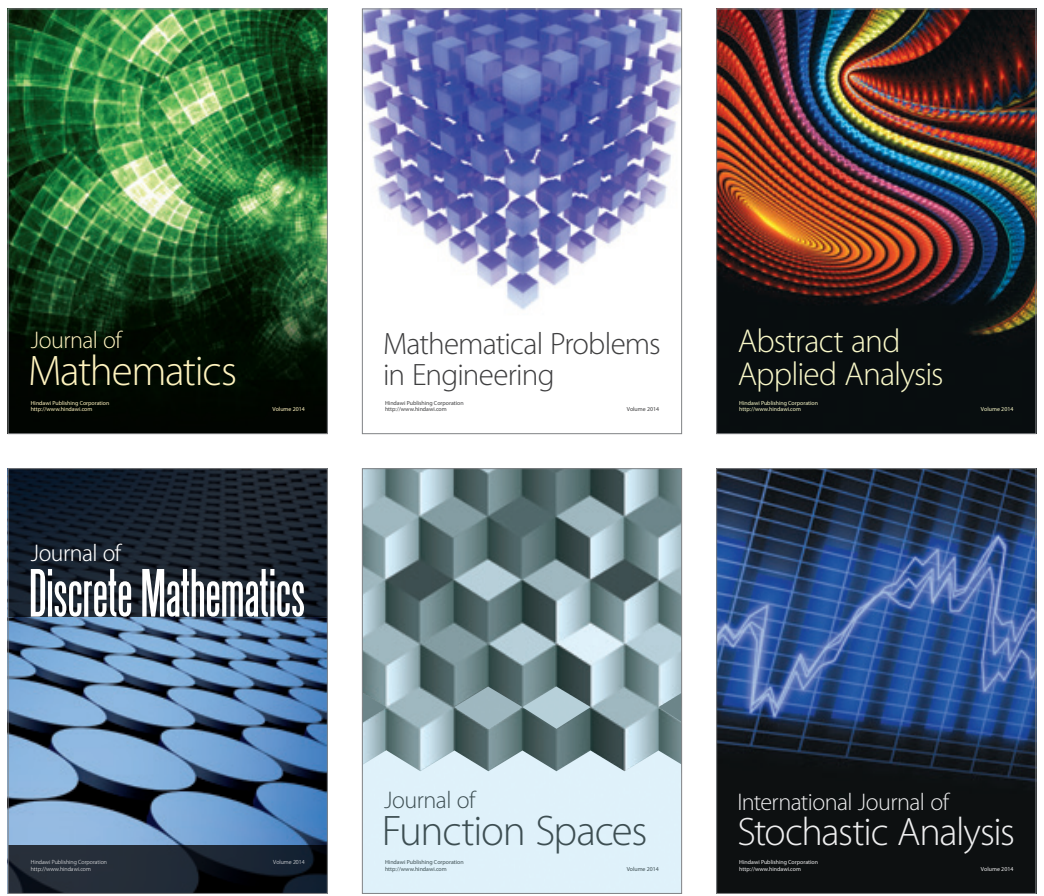

Journal of

Function Spaces

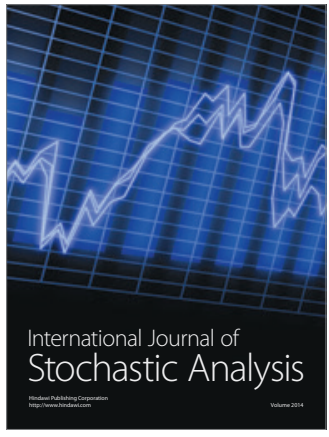

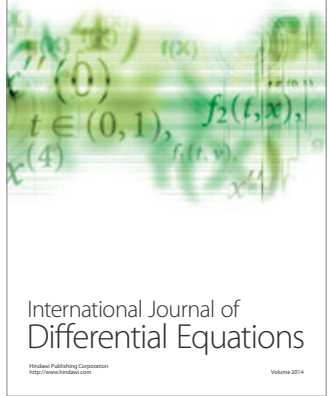
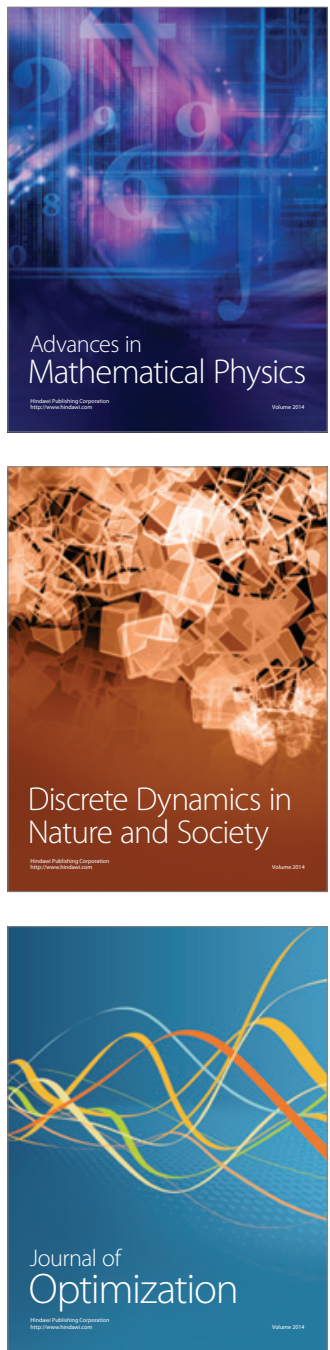